\title{
O Programa Academia da Saúde como estratégia de promoção da saúde e modos de vida saudáveis: cenário nacional de implementação
}

\author{
The Health Academy Program as a strategy to promote health \\ and healthy lifestyles: the national implementation scenario
}

\author{
Gisele Balbino Araujo Rodrigues de Sá ${ }^{1}$ \\ Gabriela Chagas Dornelles ${ }^{1}$ \\ Kátia Godoy Cruz ${ }^{1}$ \\ Roberta Corrêa de Araújo Amorim ${ }^{1}$ \\ Silvânia Suely Caribé de Araújo Andrade ${ }^{1}$ \\ Taís Porto Oliveira ${ }^{1}$ \\ Marta Maria Alves da Silva ${ }^{1}$ \\ Deborah Carvalho Malta ${ }^{1}$ \\ Maria de Fátima Marinho de Souza ${ }^{1}$
}

${ }^{1}$ Departamento de

Vigilância de Doenças e Agravos Não Transmissíveis e Promoção da Saúde. Secretaria de Vigilância em Saúde. Ministério da Saúde. SAF Sul, Trecho 02, Lotes 05 e 06, Bloco F, Torre I, Edifício Premium, Térreo, Sala 16. 70070-600 Brasília DF Brasil.gisele.rodrigues@ saude.gov.br

\begin{abstract}
The National Health Promotion Policy reasserted the Brazilian Ministry of Health's commitment to bolster the promotion of health in the Unified Health System. In this context, the Health Academy Program constitutes a new tool of the health network for the enhancement of individual and collective primary healthcare. The scope of this study is to present the program implementation scenario, describing characteristics of its operation in the country. Data were collected through an electronic form sent to all Municipal Health Departments that received federal resources to implement the program. The response rate was $85 \%$, corresponding to 2,418 municipalities. A total of 856 centers were found to be in operation, primarily promoting physical exercise, healthy eating and health education. The main participants were adults and the elderly. Difficulties reported by the administrators involve the inclusion of children and adolescents and the hiring of professionals. Over $90 \%$ of the program centers do not depend exclusively on federal funding for operation and receive municipal support to conduct their activities. The results show the potential of the program as a strategy to promote healthcare in the community nationwide in Brazil.
\end{abstract}

Key words Health promotion, Primary care, Health programs
Resumo A Política Nacional de Promoção da Saúde reafirmou o compromisso do Ministério da Saúde com o fortalecimento da promoção da saúde no Sistema Único de Saúde e, nesse contexto, o Programa Academia da Saúde se destaca como novo equipamento na rede de serviços, potencializando ações de cuidados individuais e coletivos na atenção básica. Este trabalho teve por objetivo descrever o cenário da implantação do Programa e apresentar características de seu funcionamento no país. Os dados foram coletados por meio de formulário eletrônico enviado a todas as Secretarias Municipais de Saúde que receberam recurso para implantar o Programa e a taxa de resposta foi de 85\%, correspondendo a 2418 municipios. Um total de 856 polos informou estar em funcionamento, desenvolvendo prioritariamente atividades de práticas corporais, alimentação saudável e educação em saúde. $O$ principal público participante do Programa foram adultos e idosos. Dificuldades apontadas pelos gestores incluem a inclusão de crianças e adolescentes e a contratação de profissionais. Mais de $90 \%$ dos polos não dependem exclusivamente do recurso federal para funcionamento, recebendo contrapartidas municipais para o desenvolvimento de suas atividades. Os resultados evidenciam o potencial do Programa como estratégia de promoção da saúde e produção do cuidado nas comunidades, sendo fundamental qualificar suas ações em todo o país.

Palavras-chave Promoção da saúde, Atenção Básica, Programas de saúde. 


\section{Introdução}

O conceito de Promoção da Saúde se fundamenta em uma compreensão ampla do processo saúde e doença e se constrói a partir da crítica à excessiva medicalização do setor, reconhecendo o papel das condições de vida como determinantes e condicionantes da saúde e qualidade de vida das populações ${ }^{1-3}$. Os marcos teóricos e conceituais da Promoção da Saúde remetem à I Conferência Mundial de Promoção da Saúde, realizada em Ottawa, em 1986, e preconizam a atuação integrada desse setor com outros setores para a elaboração de políticas públicas saudáveis e o empoderamento de indivíduos e coletividades para atuarem sobre fatores que afetam a sua saúde e qualidade de vida, com maior participação no controle deste processo ${ }^{4-7}$.

No Brasil, os princípios da Promoção da Saúde foram incorporados pelo Movimento da Reforma Sanitária na Constituição Federal de 1988 e na criação do Sistema Único de Saúde (SUS), com efetiva institucionalização em 2006, a partir da aprovação da Política Nacional de Promoção da Saúde (PNPS) ${ }^{8-10}$. Em 2014, em consonância com novos marcos nacionais e internacionais, a PNPS é revisada, reconhecendo a necessidade de qualificar sua operacionalização no SUS e tendo como objetivo promover a qualidade de vida e a melhoria das condições e dos modos de viver, ampliando a potencialidade da saúde individual e coletiva e reduzindo vulnerabilidades e riscos à saúde decorrentes dos determinantes sociais, econômicos, politicos, culturais e ambientais ${ }^{11,12}$.

A PNPS propõe temas prioritários a serem desenvolvidos nas diferentes esferas de governo, com a promoção da cultura de paz e direitos humanos; a promoção da mobilidade segura; a promoção do desenvolvimento sustentável; o enfrentamento do uso do tabaco e do consumo abusivo do álcool; a promoção da alimentação adequada e saudável; e a promoção de práticas corporais e atividades físicas ${ }^{12}$. Para a implementação destas prioridades, a PNPS preconiza a territorialização, a articulação intra e intersetorial, a participação e controle social, a formação profissional, entre outros eixos, como estratégias operacionais para concretizar a política nos estados e municípios.

A PNPS também aponta a necessidade de transversalizar a Promoção da Saúde na rede de atenção, favorecendo práticas de cuidado humanizadas, pautadas nas necessidades locais e na integralidade do cuidado ${ }^{12}$. Neste sentido, o Pro- grama Academia da Saúde se destaca como um novo equipamento da atenção básica com potencial de fortalecer e qualificar as ações de promoção da saúde nas comunidades ${ }^{13}$. Com estrutura e quadro de profissionais que o diferenciam das estruturas tradicionais dos serviços de saúde, o programa foi criado em 2011 visando principalmente à promoção de práticas corporais e atividades físicas sendo, porém, redefinido em 2013, com seu objetivo ampliado para contribuir para a promoção da saúde e produção do cuidado e de modos de vida saudáveis da populaçãa ${ }^{13}$.

A redefinição do Programa é um marco importante para a discussão das ações de promoção da saúde na atenção básica. Compreendido como um novo ponto de atenção na rede de serviços, o Programa se configura como uma nova porta de entrada, promovendo novas possibilidades de encontros e aproximações com os usuários. As ações do Programa articuladas com os demais serviços da rede de atenção favorecem maior integralidade nos projetos terapêuticos e promovem o estabelecimento de vínculos e corresponsabilização entre a comunidade local e os serviços.

A implantação do Programa é realizada a partir do repasse de recurso federal para a construção de espaços denominados polos, com infraestrutura e profissionais qualificados nos municí$\operatorname{pios}^{14}$. As atividades desenvolvidas no Programa devem ser culturalmente inseridas e adaptadas aos territórios, contemplando os seguintes eixos: práticas corporais e atividades físicas; produção do cuidado e de modos de vida saudáveis; promoção da alimentação saudável; práticas integrativas e complementares; práticas artísticas e culturais; educação em saúde; planejamento e gestão; e mobilização da comunidade ${ }^{13}$.

Além dos polos construídos com recurso federal, o Programa Academia da Saúde incorpora também iniciativas municipais que foram consideradas similares à proposta, e podem receber custeio do Ministério da Saúde, se adequando às suas normativas. Até maio de 2015, havia um total de 2.849 municípios contemplados com o Programa no país.Esses municípios representam um total de 4.240 polos, incluindo 450 iniciativas similares e 3790 obras em diferentes estágios de construção, destas, 1165 se encontravam finalizadas até o período deste trabalho.

Considerando a necessidade de se conhecer o cenário nacional do Programa, este estudo visa descrever as características de sua implantação e funcionamento no país no ano de 2015. 


\section{Métodos}

Trata-se de um estudo descritivo transversal. Para realização do estudo foram utilizadas informações coletadas pelo Departamento de Atenção Básica, da Secretaria de Atenção à Saúde do Ministério da Saúde (DAB/SAS) e pelo Departamento de Vigilância de Doenças e Agravos Não Transmissíveis e Promoção da Saúde, da Secretaria de Vigilância em Saúde (DANTPS/SVS), por meio de um questionário eletrônico enviado a todos os municípios contemplados com o Programa Academia da Saúde, em maio de 2015.

O questionário eletrônico consiste na estratégia de monitoramento de gestão realizada anualmente pela SVS. A coleta de dados foi realizada pelo FormSUS, um serviço de criação de formulários do ambiente DATASUS, no período de 1 a 23 de maio de 2015.

O formulário, elaborado pelo Ministério da Saúde é enviado por meio de um link às Secretarias Estaduais de Saúde (SES) que, por sua vez, o encaminham para os municípios habilitados com o Programa. Os formulários, de rápido preenchimento, são acessados e respondidos de forma online pelos responsáveis pelo Programa Academia da Saúde nos municípios, ficando imediatamente disponíveis para serem analisados pela gestão estadual e federal do Programa. As facilidades encontradas no FormSUS foram favoráveis à escolha desta ferramenta para o Ministério da Saúde, em conjunto com as SES e Secretarias Municipais de Saúde (SMS), acompanhar a implementação do Programa no país.

O formulário de monitoramento 2015 foi composto pelos seguintes blocos: dados institucionais; informações sobre o Programa; ações da Secretaria Municipal de Saúde; e sustentabilidade e fortalecimento do Programa, sendo este último respondido apenas por municípios que possuem polos em funcionamento, ou seja, desenvolvendo atividades. Além do questionário eletrônico, estas SMS que informaram possuir polos em funcionamento também responderam a uma planilha com questões relativas às atividades desenvolvidas.

Ao final do período de preenchimento, foram obtidas informações referentes a duas unidades de análise, o município como a unidade dos formulários eletrônicos e o polo como unidade de análise das planilhas de atividades. Os dados coletados foram analisados por meio de frequência absoluta e relativa, com a utilização do teste de qui-quadrado para análise comparativa entre as regiões (Centro-Oeste, Nordeste, Norte, Sudeste,
Sul). Devido à desigualdade na distribuição do número de polos em funcionamento,foi calculado um peso para ser aplicado nas análises no intuito de minimizar diferenças entre as regiões. Este peso consiste no resultado da divisão entre o número de polos em funcionamento que responderam ao questionário de monitoramento (numerador) pelo número de municípios respondentes em cada região (denominador). Foi utilizado o aplicativo STATA, versão 12.0.

\section{Resultados e discussão}

Das 2849 SMS contempladas pelo Programa, 2418 SMS responderam ao monitoramento em 2015 (Figura 1), representando 85\% dos municípios habilitados com o Programa Academia da Saúde no período (Tabela 1).

Dentre os respondentes, 1.636 municípios informaram que o Programa ainda não estava em funcionamento, em alguns casos com a construção do polo ainda não finalizada e outros com obras ainda não iniciadas. Um total de 782 SMS informou possuir polos em funcionamento e também responderam a planilha com questões relativas às atividades desenvolvidas em seus polos. Como algumas SMS possuem mais de um polo em funcionamento, o total de polos respondentes foi maior que o número de SMS respondentes, totalizando 856 polos.

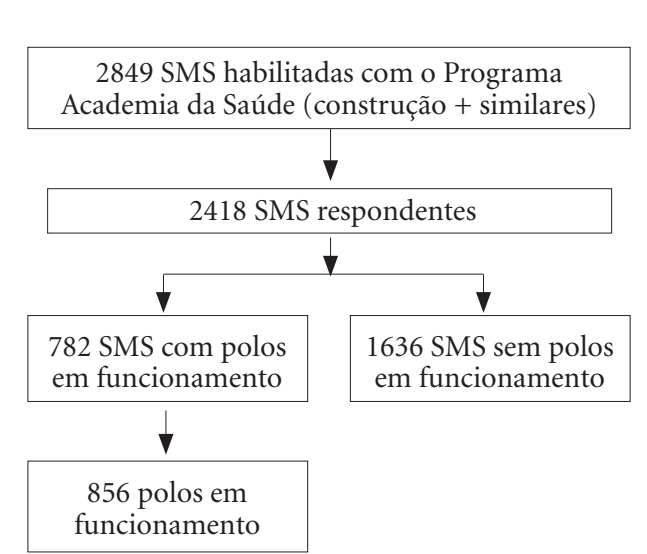

Figura 1. Fluxograma do Monitoramento Nacional do Programa Academia da Saúde em 2015. 
Tabela 1.Número de municípios habilitados com Polo do Programa Academia da Saúde e número de participantes do Monitoramento 2015 - Total e por Unidade da Federação - Brasil - Maio/2015.

\begin{tabular}{|c|c|c|c|c|c|}
\hline Região & UF & $\begin{array}{c}\text { Municípios Habilitados } \\
\text { (construção }+ \\
\left.\text { similares }^{*}\right)\end{array}$ & $\begin{array}{l}\text { Municípios que } \\
\text { responderam o } \\
\text { monitoramento }\end{array}$ & $\begin{array}{l}\text { Percentual de } \\
\text { respondentes } \\
(\%)\end{array}$ & $\begin{array}{l}\text { Número de polos } \\
\text { em funcionamento } \\
\text { respondentes }\end{array}$ \\
\hline \multirow[t]{5}{*}{ Centro-Oeste } & DF & 1 & 1 & 100 & 0 \\
\hline & $\mathrm{GO}$ & 125 & 120 & 96 & 35 \\
\hline & MS & 63 & 42 & 67 & 12 \\
\hline & MT & 67 & 61 & 91 & 12 \\
\hline & Subtotal & 256 & 224 & 8 & 59 \\
\hline \multirow[t]{10}{*}{ Nordeste } & $\mathrm{AL}$ & 57 & 50 & 88 & 9 \\
\hline & BA & 200 & 168 & 84 & 55 \\
\hline & $\mathrm{CE}$ & 113 & 108 & 96 & 33 \\
\hline & MA & 91 & 60 & 66 & 15 \\
\hline & $\mathrm{PB}$ & 155 & 143 & 92 & 45 \\
\hline & $\mathrm{PE}$ & 135 & 132 & 98 & 144 \\
\hline & PI & 107 & 82 & 77 & 19 \\
\hline & $\mathrm{RN}$ & 92 & 80 & 87 & 23 \\
\hline & SE & 45 & 45 & 100 & 18 \\
\hline & Subtotal & 995 & 868 & 30 & 361 \\
\hline \multirow[t]{8}{*}{ Norte } & $\mathrm{AC}$ & 22 & 22 & 100 & 10 \\
\hline & $\mathrm{AM}$ & 13 & 9 & 69 & 2 \\
\hline & $\mathrm{AP}$ & 11 & 6 & 55 & 0 \\
\hline & $\mathrm{PA}$ & 94 & 69 & 73 & 23 \\
\hline & RO & 12 & 12 & 100 & 2 \\
\hline & $\mathrm{RR}$ & 12 & 11 & 92 & 7 \\
\hline & TO & 93 & 51 & 55 & 17 \\
\hline & Subtotal & 257 & 180 & 6 & 61 \\
\hline \multirow[t]{5}{*}{ Sudeste } & ES & 30 & 24 & 80 & 8 \\
\hline & MG & 376 & 319 & 85 & 137 \\
\hline & $\mathrm{RJ}$ & 60 & 51 & 85 & 13 \\
\hline & SP & 272 & 226 & 83 & 78 \\
\hline & Subtotal & 738 & 620 & 22 & 236 \\
\hline \multirow[t]{5}{*}{ Sul } & $\mathrm{PR}$ & 197 & 163 & 83 & 60 \\
\hline & RS & 282 & 239 & 85 & 34 \\
\hline & SC & 124 & 124 & 100 & 45 \\
\hline & Subtotal & 603 & 526 & 18 & 139 \\
\hline & Brasil & 2849 & 2418 & 85 & 856 \\
\hline
\end{tabular}

*Além dos polos construídos com recurso federal, o Programa Academia da Saúde incorpora também iniciativas municipais que foram consideradas similares à proposta, e podem receber custeio do Ministério da Saúde, se adequando às suas normativas.

Fonte: Ministério da Saúde, Secretaria de Vigilância em Saúde, Coordenação Geral das Doenças e Agravos Não Transmissíveis e Promoção da Saúde - Monitoramento 2015 Programa Academia da Saúde.

\section{Características de Implantação}

Os resultados do monitoramento mostraram que quase $70 \%(\mathrm{n}=1.636)$ dos municípios respondentes ainda não possuíam polos do Programa desenvolvendo atividades, em alguns casos a construção do polo ainda não estava finalizada e em outros as obras ainda não haviam sido iniciadas.
Dificuldades na gestão da construção de obras públicas são comuns na maioria dos municípios brasileiros, segundo Neiva e Camacho ${ }^{15}$ a falta de planejamento, de fiscalização e as fraudes são apontadas pelos relatórios dos órgãos de controle como as principais causas do desperdício de recursos, do alto número de obras inacabadas e de construções de má qualidade nos municípios do Brasil. Além disso, em muitos casos, falta 
competência técnica nas prefeituras e secretarias municipais para a gestão das etapas que envolvem a construção de estruturas físicas - estudos de viabilidade, escolha de terreno, elaboração de projeto arquitetônico, e outras ${ }^{16}$. Em 2014, o Ministério da Saúde realizou um inquérito telefônico junto aos municípios que haviam recebido recurso de construção do Programa Academia da Saúde no ano de 2011 e que ainda não o possuíam implantado; os gestores informaram que problemas com licitação, mudanças de prefeito e insuficiência de recursos foram os principais motivos para o atraso ou a impossibilidade de implantação ${ }^{17}$. Deste modo, a plena implantação do Programa Academia da Saúde nos territórios segue como um grande desafio para a gestão em todos os níveis de governo, sendo necessárias estratégias para evitar a devolução de recursos e garantir que os municípios possuam o programa em sua rede de serviços.

Em relação à articulação do programa com outros serviços da atenção básica, o monitoramento indicou que $68 \%(\mathrm{n}=1.644)$ dos municípios habilitados possuíam Núcleos de Apoio à Saúde da Família (NASF) implantados, porém, dentre os municípios com polos em funcionamento, 30\% informaram que o Programa não estava vinculado ao NASF no território, sinalizando dificuldades para ações integradas. Por outro lado, apenas $7 \%$ dos 856 polos em funcionamento informaram não receber nenhum tipo de apoio de outros profissionais da atenção básica. A necessidade de superação da fragmentação de ações nos serviços de saúde, incluindo as equipes de atenção básica, tem sido discutida por diversos autores ${ }^{18,19}$. Segundo a Portaria MS no 2.681, de 7 de novembro de 2013, o Programa Academia da Saúde deve configurar-se como ponto de atenção da Rede de Atenção à Saúde, complementar e potencializador das ações de cuidados individuais e coletivos na atenção básica. Deste modo, é fundamental que suas ações estejam integradas com os demais serviços, particularmente da atenção básica, com vistas à construção de projeto assistencial comum ${ }^{13}$. Os resultados evidenciam a importância de estratégias que contribuam para a organização do processo de trabalho de forma mais integral e resolutiva, fortalecendo e qualificando as ações de promoção da saúde no território. A interlocução da equipe responsável pelo Programa Academia da Saúde com as das Unidades Básicas de Saúde (UBS), da Estratégia de Saúde da Família (ESF) e dos NASF possibilita o estabelecimento de prioridades adequadas à realidade local e a construção de linhas de cuidado que efetivamente atendam às necessidades dos usuários, contribuindo para uma maior integralidade e continuidade dos serviços.

\section{Polos em funcionamento}

Ao todo, 782 municípios informaram já estar com atividades em andamento e, dentre estes, 856 polos que já desenvolvem atividades enviaram suas informações, analisadas a seguir.

\section{Perfil dos participantes das atividades}

Quase $100 \%$ dos polos informaram desenvolver atividades com idosos e adultos e 76\% (n $=647$ ) dos respondentes receberam adolescentes em suas atividades. Apenas 38\% $(\mathrm{n}=328)$ dos polos responderam incluir crianças e 36\% ( $\mathrm{n}=$ 306) informaram a participação de pessoas em todas as faixas etárias nas atividades (Tabela 2).

Muitos programas de educação e promoção da saúde ofertados nas UBS são direcionados a indivíduos idosos ou adultos, sendo pouco atrativos para jovens, crianças e mesmo para adultos e idosos saudáveis. É comum que essas atividades sejam organizadas em grupos com enfoque em doenças crônicas ou condições específicas, como hipertensão, diabetes, tabagismo, gravidez na adolescência, etc. ${ }^{20,21}$. Nesse sentido, o Programa Academia da Saúde, ao preconizar uma perspectiva positiva, com oferta de atividades para pessoas nos diferentes ciclos de vida - crianças, adolescentes, jovens, adultos e idosos - independentemente de sua condição de saúde, tem o potencial de ampliar a participação de grupos que, de modo geral, não são captados pelos serviços de saúde.

O trabalho na perspectiva dos ciclos de vida reconhece as especificidades biológicas, sociais e culturais de cada etapa de desenvolvimento, o que torna possível a construção de projetos terapêuticos mais adequados à realidade dos usuários ${ }^{22-24}$. No entanto, o monitoramento indicou que em $56 \%$ dos polos as equipes têm dificuldades para desenvolver atividades com crianças e $41 \%$ atividades com adolescentes (Tabela 2). Este resultado reflete a necessidade de ações de educação permanente para capacitar profissionais para o trabalho com esses grupos específicos, favorecendo o acolhimento adequado de crianças e adolescentes em atividades apropriadas, contribuindo para o estabelecimento de vínculo entre eles e o serviço. Conforme apontado por Ferrari et al. ${ }^{25}$, a relativa ausência dos adolescentes nos serviços de saúde se deve em parte a pouca oferta de ações direcionadas a eles e em parte à baixa procura pelos mesmos, fatores que estão interligados e 
Tabela 2. Caracterização do funcionamento dos polos do Programa Academia da Saúde - Brasil, 2015.

\begin{tabular}{|c|c|c|}
\hline Atividades & Número de Municípios & Percentual de Municípios (\%) \\
\hline \multicolumn{3}{|l|}{ Turno de atividade } \\
\hline Manhã & 798 & 93 \\
\hline Tarde & 728 & 85 \\
\hline Noite & 384 & 45 \\
\hline Todos & 287 & 34 \\
\hline \multicolumn{3}{|l|}{ Participantes das atividades } \\
\hline Idosos & 847 & 99 \\
\hline Adultos & 847 & 99 \\
\hline Adolescentes & 647 & 76 \\
\hline Crianças & 328 & 38 \\
\hline Todos & 306 & 36 \\
\hline \multicolumn{3}{|l|}{ Atividades desenvolvidas nos polos } \\
\hline Práticas corporais & 825 & 96 \\
\hline Promoção da Alimentação Saudável & 779 & 91 \\
\hline Práticas integrativas e complementares & 488 & 57 \\
\hline Práticas artísticas & 248 & 29 \\
\hline Educação em Saúde & 805 & 94 \\
\hline Mobilização da Comunidade & 753 & 88 \\
\hline \multicolumn{3}{|l|}{ Vínculo empregatício dos profissionais } \\
\hline Efetivo & 419 & 49 \\
\hline Contrato temporário & 608 & 71 \\
\hline CLT & 128 & 15 \\
\hline Cedido & 171 & 20 \\
\hline Outros & 77 & 9 \\
\hline \multicolumn{3}{|l|}{ Contrapartida municipal } \\
\hline Aquisição material consumo & 745 & 87 \\
\hline Aquisição material permanente & 710 & 83 \\
\hline Manutenção Polo & 762 & 89 \\
\hline Contratação profissionais excedentes & 317 & 37 \\
\hline Construção complementar & 274 & 32 \\
\hline Outros & 43 & 5 \\
\hline Não recebe & 68 & 8 \\
\hline
\end{tabular}

Fonte: Ministério da Saúde, Secretaria de Vigilância em Saúde, Coordenação Geral das Doenças e Agravos Não Transmissíveis e Promoção da Saúde - Monitoramento 2015 Programa Academia da Saúde.

intimamente relacionados à forma como o serviço de saúde está estruturado. Nesse sentido, é válido ressaltar a intergeracionalidade como um dos princípios do Programa a ser explorado na estruturação das atividades, com a valorização do diálogo e da troca de saberes entre gerações ${ }^{13,26}$.

A respeito da inclusão de minorias no Programa, o monitoramento mostrou que em $90 \%$ dos municípios $(\mathrm{n}=708)$ as atividades oferecidas pelo Programa contemplam apenas a comunidade em geral. Um percentual baixo de municípios informou a inclusão de quilombolas $(3,2 \%, \mathrm{n}=$ $25)$, ribeirinhos $(2,3 \%, \mathrm{n}=18)$, indígenas $(2 \%, \mathrm{n}$ $=16)$, pessoas em situação de rua $(1,4 \%, \mathrm{n}=11)$ e ciganos $(0,6 \%, n=5)$ em suas atividades. Al- guns respondentes $(5 \%, \mathrm{n}=43)$ afirmaram não haver nenhum desses grupos no município. Destaca-se a necessidade de reflexão sobre formas de se identificar populações específicas que não estão sendo contempladas pelas atividades oferecidas, pois, nos municípios brasileiros, dificilmente não serão encontrados pessoas em situação de rua, indígenas, ribeirinhos, ciganos, quilombolas ou outros. É fundamental buscar compreender as barreiras para a participação de grupos historicamente excluídos. Como parte da rede de serviços do SUS, o Programa Academia da Saúde deve ser socialmente inclusivo, não reproduzindo em seus espaços e práticas a marginalização de grupos em situação de maior vulnerabilidade social. É ne- 
cessário planejar e desenvolver estrategicamente as atividades do polo de modo a ampliar seu alcance e garantir maior equidade.

\section{Características da oferta de atividades}

No que diz respeito às atividades ofertadas pelo Programa, 93\% dos polos $(\mathrm{n}=798)$ informaram oferecer atividades pela manhã, $85 \%$ (n $=728)$ no período da tarde, $45 \%(\mathrm{n}=384)$ desenvolveram atividades à noite e $34 \%(n=287)$ em todos os turnos (Tabela 2). Sabe-se que os horários de funcionamento dos serviços de saúde possuem impacto direto no acesso de determinados grupos, como trabalhadores, que ficam impossibilitados de usufruir do serviço em função das suas atividades serem predominantemente diurnas ${ }^{27}$. Os resultados do monitoramento indicam um cenário positivo quanto à oferta de atividades, com quase metade dos polos com funcionamento noturno e um terço em tempo integral. As características da estrutura física e a natureza das atividades oferecidas pelo Programa Academia da Saúde podem ser fatores que favorecem a oferta de horários alternativos, e fatores como clima e temperatura também podem influenciar a escolha dos turnos de funcionamento nas diferentes regiões do país. O resultado também enfatiza o potencial do Programa enquanto um equipamento que se diferencia dos serviços tradicionais da atenção básica, com oferta de atividades alternativas, que o tornam uma importante estratégia de ampliação do acesso aos serviços de saúde nas comunidades.

Quanto aos tipos de atividades desenvolvidas pelo Programa, 96\% $(n=825)$ dos polos informaram oferecer práticas corporais e atividades físicas, 94\% (n-805) desenvolveram atividades de educação em saúde, e 91\% ( $\mathrm{n}=775)$ realizaram ações de alimentação saudável. Práticas integrativas e complementares em saúde(PICS) foram ofertadas em $57 \%(\mathrm{n}=489)$ dos polos e práticas artísticas por apenas $29 \%(\mathrm{n}=250)$ dos respondentes (Tabela 2). Este resultado dialoga com os eixos preconizados pela portaria do Programa e evidencia o predomínio das práticas corporais, de promoção da alimentação saudável e de educação em saúde no cotidiano dos polos. São necessários estudos para explorar as características das atividades oferecidas, no sentido de qualificá-las conforme os princípios e valores definidos na PNPS ${ }^{28-31}$. Além disso, os dados apontam a necessidade de fortalecer a oferta de outras atividades, como PICS e práticas artísticas, temas que podem ser incorporados nas ações de capacita- ção e educação permanente ${ }^{32-35}$. Recomenda-se também a incorporação de mecanismos de escuta para identificar os interesses dos usuários e incorporá-los na oferta de atividades, com a construção de uma agenda que leve em consideração os valores socioculturais, as vulnerabilidades e as necessidades de saúde das comunidades.

\section{Sustentabilidade do Programa}

A sustentabilidade de projetos costuma ser definida como a capacidade de uma intervenção proporcionar benefícios sem interrupção durante um longo período de tempo ${ }^{36}$. Nesse sentido, aspectos como institucionalização, vínculo empregatício dos profissionais e recebimento de contrapartidas municipais foram analisados como fatores que indicam maior ou menor grau de sustentabilidade do Programa nos territórios.

Segundo o monitoramento, $86 \%$ dos municípios informaram ter incluído o Programa no Plano Municipal de Saúde, resultado favorável em termos de sustentabilidade local, visto que o Plano de Saúde induz o direcionamento de recursos e o monitoramento de resultados, além de promover o controle social ${ }^{37}$.

Em relação ao vínculo empregatício, os dados do Programa mostraram que em $71 \%(n=608)$ dos polos os profissionais estão vinculados por meio de contrato temporário de prestação de serviços e apenas $49 \%(n=419)$ possuem profissionais efetivos atuando em suas atividades, particularmente nos municípios que já possuíam iniciativas similares anteriores ao Programa Academia da Saúde, como Recife e Belo Horizonte (Tabela 2). Outras formas de vínculo empregatício também foram informadas, $20 \%(\mathrm{n}=173)$ dos polos possuem profissionais cedidos de outras secretarias, $15 \%(\mathrm{n}=126)$ funcionam com celetistas e $9 \%(\mathrm{n}=77)$ informaram ter profissionais com cargos comissionados, estagiários e voluntários (Tabela 2).O vínculo empregatício se remete à regulação das relações de trabalho e deve dar suporte ao bom desenvolvimento do mesmo. $\mathrm{Na}$ saúde as dificuldades encontradas para a ampliação e fixação dos quadros de pessoal são notáveis e se constituem como um dos principais desafios de gestão do setor ${ }^{38}$, sendo também um desafio para a sustentabilidade do Programa.

No que diz respeito ao recebimento de contrapartidas municipais complementares ao recurso de custeio federal para manutenção das atividades do programa, o monitoramento mostrou que $89 \%(\mathrm{n}=764)$ dos polos receberam recurso municipal para a manutenção do espaço, $87 \%$ 
( $\mathrm{n}=747)$ para a aquisição de materiais de consumo e $83 \%(n=707)$ para adquirir materiais permanentes. Um percentual relativamente baixo, $8 \%(n=66)$ dos polos, não recebe nenhuma contrapartida municipal e apenas $37 \%(n=315)$ recebem apoio para a contratação de profissionais. Esse foi um resultado positivo, que sinaliza a incorporação do Programa Academia da Saúde na agenda de prioridades das SMS (Tabela 2).

\section{Diferenças por região}

Foram encontradas diferenças estatisticamente significativas na região Nordeste para as seguintes variáveis: oferta de atividade no turno da noite; participação de adolescentes; participação de idosos; realização de práticas corporais; realização de atividades de alimentação saudável; mobilização da comunidade; profissionais com vínculo efetivo; profissionais com contrato temporário; celetistas; profissionais cedidos de outras secretarias; material de consumo como contrapartida municipal; manutenção do polo como contrapartida municipal; contratação de profissionais como contrapartida municipal (Tabela 3). Uma hipótese para este resultado é que a região possui um número maior de polos que foram habilitados como similares e, portanto, se encontravam em funcionamento desde o início da implantação do Programa, em 2011. Nas demais regiões, houve predomínio da habilitação ao Programa para a construção de polos, em diversos casos, com dificuldades para implementação e, consequentemente, para o funcionamento dos mesmos. Apesar da atribuição do peso ter buscado minimizar a influência da distribuição desigual de polos em funcionamento nas diferentes regiões do país, é possível que haja efeito residual atribuído ao maior número de polos funcionando na região Nordeste.

\section{Conclusão}

O monitoramento do Programa Academia da Saúde é uma importante ferramenta de gestão que permite identificar suas potencialidades e fragilidades, subsidiando a tomada de decisão nos diferentes níveis de gestão. O cenário descrito neste trabalho evidencia a necessidade de apoio à implementação do programa nas SMS que ainda apresentam um grande número de obras não finalizadas ou não iniciadas. Já nos locais com atividades em funcionamento, destaca-se a ampla oferta de práticas corporais, ações de promoção da alimentação saudável e ações de educação em saúde, com oferta ainda incipiente de práticas integrativas e complementares e artísticas. Ressalta-se ainda que, além da participação de adultos e idosos, o Programa ainda pouco incorpora adolescentes, crianças e pessoas em situação de maior vulnerabilidade (pessoas em situação de rua, quilombolas, ribeirinhos, e outros) em suas atividades, sendo necessário fortalecer e qualificar a oferta de atividades para esses grupos.

A presença do Programa Academia da Saúde no plano municipal da maioria das SMS constitui cenário favorável à sua sustentabilidade no território, com a maioria dos polos recebendo apoio municipal para seu funcionamento. Faz-se necessário, porém, uma maior articulação entre os três níveis de gestão para apoio à implementação das ações dos polos e fortalecimento do programa. Nesse sentido, destaca-se que intervenções de promoção da saúde, como é o caso do Programa Academia da Saúde, visam impactos de longo prazo e é importante definir estratégias para assegurar sua sustentabilidade na agenda local.

Os resultados apresentados evidenciam o potencial do Programa como estratégia de promoção da saúde e produção do cuidado nas comunidades, sendo fundamental a qualificação de suas ações em consonância com os princípios e valores da PNPS. 
Tabela 3. Percentual de polos, por região, segundo as características de funcionamento, Brasil, 2015.

\begin{tabular}{|c|c|c|c|c|c|c|c|c|c|}
\hline \multirow{2}{*}{ Atividades } & & \multicolumn{7}{|c|}{ Percentual de Polos por Região } & \multirow{2}{*}{$\begin{array}{l}\text { Valor } \\
\text { de p }\end{array}$} \\
\hline & & $\mathrm{CO}$ & $\mathrm{NE}$ & $\mathrm{N}$ & $\mathrm{CO}$ & SE & $S$ & Total & \\
\hline \multicolumn{10}{|l|}{ Turno de atividade } \\
\hline \multirow[t]{2}{*}{ Manhã } & $\operatorname{Sim}$ & 4,37 & 45,72 & 5,85 & 4,37 & 26,89 & 10,72 & 93,55 & 0,108 \\
\hline & Não & 0,59 & 0,25 & 0,76 & 0,59 & 0,17 & 1,01 & 6,45 & \\
\hline \multirow[t]{2}{*}{ Tarde } & Sim & 4,12 & 39,98 & 5,74 & 4,12 & 24,11 & 10,72 & 84,67 & 0,266 \\
\hline & Não & 0,84 & 8,13 & 0,87 & 0,84 & 4,48 & 1,01 & 15,33 & \\
\hline \multirow[t]{2}{*}{ Noite } & $\operatorname{Sim}$ & 2,44 & 26,12 & 2,06 & 2,44 & 10,9 & 4,22 & 45,74 & 0,000 \\
\hline & Não & 2,52 & 21,99 & 4,55 & 2,52 & 17,69 & 7,51 & 54,26 & \\
\hline \multicolumn{10}{|l|}{ Participantes das atividades } \\
\hline \multirow[t]{2}{*}{ Idosos } & Sim & 4,79 & 47,45 & 6,28 & 4,79 & 28,47 & 11,64 & 98,63 & 0,049 \\
\hline & Não & 0,17 & 0,67 & 0,33 & 0,17 & 0,12 & 0,084 & 1,37 & \\
\hline \multirow[t]{2}{*}{ Adultos } & Sim & 4,88 & 47,58 & 6,61 & 4,88 & 28,47 & 11,31 & 98,84 & 0,098 \\
\hline & Não & 0,084 & 0,53 & 0 & 0,084 & 0,12 & 0,42 & 1,16 & \\
\hline \multirow[t]{2}{*}{ Adolescentes } & Sim & 3,45 & 38,78 & 5,09 & 3,45 & 19,38 & 9,11 & 75,82 & 0,002 \\
\hline & Não & 1,51 & 9,33 & 1,52 & 1,51 & 9,21 & 2,62 & 24,18 & \\
\hline \multirow[t]{2}{*}{ Crianças } & Sim & 2,19 & 19,33 & 2,82 & 2,19 & 8,96 & 4,81 & 38,1 & 0,097 \\
\hline & Não & 2,77 & 28,7 & 3,79 & 2,77 & 19,62 & 6,92 & 61,9 & \\
\hline \multicolumn{10}{|l|}{ Atividades desenvolvidas nos polos } \\
\hline \multirow[t]{2}{*}{ Práticas corporais } & Sim & 4,79 & 46,91 & 6,28 & 4,79 & 28,1 & 10,63 & 96,73 & 0,002 \\
\hline & Não & 0,17 & 1,2 & 0,33 & 0,17 & 0,48 & 1,1 & 3,27 & \\
\hline \multirow{2}{*}{ Promoção da Alimentação Saudável } & Sim & 4,2 & 44,65 & 6,18 & 4,2 & 24,59 & 10,97 & 90,05 & 0,011 \\
\hline & Não & 0,76 & 3,47 & 0,43 & 0,76 & 4 & 0,76 & 9,41 & \\
\hline \multirow[t]{2}{*}{ Práticas integrativas e complementares } & Sim & 3,11 & 25,59 & 3,36 & 3,11 & 17,69 & 7 & 56,75 & 0,117 \\
\hline & Não & 1,85 & 22,52 & 3,25 & 1,85 & 10,9 & 4,73 & 43,25 & \\
\hline Práticas artísticas & Sim & 1,26 & 13,73 & 1,73 & 1,26 & 9,21 & 3,38 & 29,3 & 0,745 \\
\hline & Não & 3,7 & 34,39 & 4,88 & 3,7 & 19,38 & 8,35 & 70,7 & \\
\hline Educação em Saúde & Sim & 4,54 & 45,85 & 6,39 & 4,54 & 26,53 & 10,88 & 94,19 & 0,435 \\
\hline & Não & 0,42 & 2,27 & 0,22 & 0,42 & 2,06 & 0,84 & 5,81 & \\
\hline Mobilização da Comunidade & $\operatorname{Sim}$ & 4,2 & 44,38 & 6,07 & 4,2 & 23,26 & 10,04 & 87,95 & 0,000 \\
\hline & Não & 0,76 & 3,73 & 0,54 & 0,76 & 5,33 & 1,69 & 12,05 & \\
\hline Vínculo empregatício dos profissionais & & & & & & & & & \\
\hline Efetivo & Sim & 2,77 & 17,99 & 2,28 & 2,77 & 15,87 & 8,35 & 47,26 & 0,000 \\
\hline & Não & 2,19 & 30,12 & 4,33 & 2,19 & 12,72 & 3,38 & 52,74 & \\
\hline Contrato temporário & Sim & 4,29 & 38,25 & 6,28 & 4,29 & 15,26 & 7,26 & 71,34 & 0,000 \\
\hline & Não & 0,67 & 9,86 & 0,33 & 0,67 & 13,32 & 4,47 & 28,66 & \\
\hline CLT & $\operatorname{Sim}$ & 0,5 & 2,67 & 0,33 & 0,5 & 7,87 & 2,7 & 14,07 & 0,000 \\
\hline & Não & 4,46 & 45,45 & 6,28 & 4,46 & 20,71 & 9,03 & 85,93 & \\
\hline Cedido & Sim & 1,01 & 9,06 & 0,65 & 1,01 & 5,57 & 3,46 & 19,75 & 0,027 \\
\hline & Não & 3,95 & 39,05 & 5,96 & 3,95 & 23,02 & 8,27 & 80,25 & \\
\hline Outros & Sim & 0,17 & 1,47 & 0,22 & 0,17 & 6,3 & 0,68 & 8,82 & 0,000 \\
\hline & Não & 4,79 & 46,65 & 6,39 & 4,79 & 22,29 & 11,05 & 91,18 & \\
\hline Contrapartida mur & & & & & & & & & \\
\hline Aquisição material consumo & Sim & 4,37 & 41,18 & 5,42 & 4,37 & 26,41 & 9,96 & 87,34 & 0,042 \\
\hline & Não & 0,59 & 6,93 & 1,19 & 0,59 & 2,18 & 1,77 & 12,66 & \\
\hline Aquisição material permanente & Sim & 4,2 & 38,38 & 5,42 & 4,2 & 24,71 & 9,7 & 82,42 & 0,235 \\
\hline & Não & 0,76 & 9,73 & 1,19 & 0,76 & 3,88 & 2,03 & 17,58 & \\
\hline Manutenção Polo & Sim & 4,46 & 41,18 & 5,74 & 4,46 & 27,38 & 10,38 & 89,14 & 0,000 \\
\hline & Não & 0,5 & 6,93 & 0,87 & 0,5 & 1,21 & 1,35 & 10,86 & \\
\hline Contratação profissionais excedentes & Sim & 2,02 & 14,79 & 2,6 & 2,02 & 13,57 & 3,71 & 36,69 & 0,000 \\
\hline & Não & 2,94 & 33,32 & 4,01 & 2,94 & 15,02 & 8,02 & 63,31 & \\
\hline Construção complementar & Sim & 1,43 & 14,53 & 1,41 & 1,43 & 10,9 & 3,46 & 31,73 & 0,052 \\
\hline & Não & 3,53 & 33,59 & 5,2 & 3,53 & 17,69 & 8,27 & 68,27 & \\
\hline Outro tipo de contrapartida & Sim & 0,25 & 1,87 & 0,43 & 0,25 & 1,94 & 0,42 & 4,91 & 0,413 \\
\hline & Não & 4,71 & 46,25 & 6,18 & 4,71 & 26,65 & 11,31 & 95,09 & \\
\hline Não recebe & Sim & 0,5 & 4,93 & 0,76 & 0,5 & 0,85 & 0,76 & 7,8 & 0,005 \\
\hline & Não & 4,46 & 43,18 & 5,85 & 4,46 & 27,74 & 10,97 & 92,2 & \\
\hline
\end{tabular}

Fonte: Ministério da Saúde, Secretaria de Vigilância em Saúde, Coordenação Geral das Doenças e Agravos Não Transmissíveis e Promoção da Saúde - Monitoramento 2015 Programa Academia da Saúde. 


\section{Colaboradores}

GBAR de Sá, GC Dornelles, KG Cruz e RCA Amorim trabalharam na coleta, análise e interpretação dos dados e na elaboração do artigo. TP Oliveira participou da elaboração do artigo. SSCA Andrade trabalhou na análise e interpretação dos dados e participou da elaboração do artigo. MMA Silva, DC Malta e MFM de Souza foram responsáveis pela revisão crítica do estudo e aprovação da versão a ser publicada.

\section{Referências}

1. Sicoli JL, Nascimento PR. Promoção de saúde: concepções, princípios e operacionalização. Interface (Botucatu) 2003; 7(12):101-122.

2. Heidmann ITSB, Almeida MCP, Boehs AE, Wosny AM, Monticelli M. Promoção à saúde: trajetória histórica de suas concepções. Texto Contexto Enferm 2006; 15(2):352-358.

3. Buss PM. Uma introdução ao conceito de promoção da saúde. In: Czeresnia D, Freitas CM, organizadores. Promoção da saúde: conceitos, reflexões, tendências. Rio de Janeiro: Fiocruz; 2003.

4. World Health Organization (WHO). The Ottawa charter for health promotion. Geneve: WHO; 1986.

5. Brasil. Ministério da Saúde (MS). As cartas de promoção à saúde. Brasília: MS; 2002.

6. Buss PM. Promoção da saúde e qualidade de vida. Cien Saude Colet 2000; 5(1):163-177.

7. Carvalho SR. Os múltiplos sentidos da categoria empowerment no projeto de promoção à saúde. Cad Saude Publica 2004; 20(4):1088-1095.

8. Brasil. Ministério da Saúde (MS). Política Nacional de Promoção da Saúde. Brasília: MS; 2006.

9. Buss PM, Carvalho AI. Desenvolvimento da promoção da saúde no Brasil nos últimos vinte anos (19882008). Cien Saude Colet 2009; 14(6):2305-2316.

10. Malta DC, Silva MMAS, Albuquerque GM, Lima CM, Cavalcante T, Jaime PC,Silva Júnior JB. A implementação das prioridades da Política Nacional de Promoção da Saúde, um balanço, 2006 a 2014. Cien Saude Colet 2014; 19(11):4301-4311.

11. Rocha DG, Alexandre VP, Marcelo VC, Rezende R, Nogueira JD, Sá RF. Processo de revisão da Política Nacional de Promoção da Saúde: múltiplos movimentos simultâneos. Cien Saude Colet 2014; 19(11):4313-4322.

12. Brasil. Portaria no 2446, de 11 de novembro de 2014 Redefine a Política Nacional de Promoção da Saúde (PNPS). Diário Oficial da União 2014; 13 nov.

13. Brasil. Portaria no 2681 , de 7 de novembro de 2013. Redefine o Programa Academia da Saúde no âmbito do Sistema Único de Saúde (SUS). Diário Oficial da União 2013; 8 nov.

14. Brasil. Portaria no 2684, de 8 de novembro de 2013. Redefine as regras e os critérios referentes aos incentivos financeiros de investimento para construção de polos e de custeio no âmbito do Programa Academia da Saúde e os critérios de similaridade entre Programas em Desenvolvimento no Distrito Federal ou no Município e o Programa Academia da Saúde. Diário Oficial da União 2013; 8 nov. 
15. Neiva AAV, Camacho SMG. Controladoria Geral do Estado da Paraíba. Controles internos na etapa de elaboração de projeto básico no sistema de produção de obras públicas. In: XI Simpósio Nacional de Auditoria de Obras Públicas; 2006; João Pessoa.

16. Castro LC, Abdalla JGF, Hippert MAS. A gestão do projeto arquitetônico em órgãos públicos: estudo de caso em unidades de saúde na prefeitura de Juiz de Fora. In: Encontro Nacional de Tecnologia do Ambiente Construído; 2012; Juiz de Fora.

17. Mielke GI, organizador. Documento Técnico contendo análise dos dados do inquérito telefônico com gestores municipais de saúde sobre a implantação do Programa Academia da Saúde em municípios custeados pelo Ministério da Saúde nos anos de 2011 e 2012. Brasília: Ministério da Saúde; 2015.

18. Giovanella L, Escorel S, Mendonça MHM. Porta de entrada pela atenção básica? Integração do PSF à rede de serviços de saúde. Saude Debate 2003; 27(65):278-289.

19. Silva IZQJ, Trad LAB. O trabalho em equipe no PSF: investigando a articulação técnica e a interação entre os profissionais. Interface (Botucatu) 2005; 9(16):25-38

20. Carvalho, YM. Atividade física e Saúde: Onde está e quem é o "Sujeito" da relação. Rev Bras Cienc Esporte 2001; 22(2):9-21.

21. Dias VP, Silveira DT, Witt RR. Educação em Saúde: o trabalho de grupos em atenção primária. Revista APS 2009; 12(2):221-227.

22. Oliveira MK. Ciclos de vida: algumas questões sobre a psicologia do adulto. Educ Pesqui 2004; 30(2):211-229.

23. Brasil. Ministério da Saúde (MS). Acolhimento à Demanda Espontânea. Brasília: MS; 2013.

24. Diba D, D'oliveira AF. Teatro e comunidade, juventude e apoio social: atores da promoção da saúde. Cien Saude Colet 2015; 20(5):1353-1362.

25. Ferrari RAP, Thomson Z, Melchior R. Atenção à saúde dos adolescentes: percepção dos médicos e enfermeiros das equipes da saúde da família. Cad Saude Publica 2006; 22(11):2491-2495.

26. Spanhol C, Lima FD, Lima M. Transmissão intergeracional: uma contribuição ao estudo do comportamento do consumidor de alimentos. Contextus - Revista Contemporânea de Economia e Gestão 2011; 8(2):31-40.

27. Cunha ABO, Vieira-da-Silva LM. Acessibilidade aos serviços de saúde em um município do Estado da Bahia, Brasil, em gestão plena do sistema. Cad Saude Publica 2010; 26(4):725-737.

28. Alves VS. Um modelo de educação em saúde para o Programa Saúde da Família: pela integralidade da atenção e reorientação do modelo assistencial. Interface (Botucatu) 2005; 9(16):39-52.
29. Castro A, Malo M, organizadores. SUS: ressignificando a promoção da saúde. São Paulo: Hucitec, Opas; 2006.

30. Freitas FF, Brasil FK, Silva CL. Práticas corporais e saúde: novos olhares. Rev Brasileira de Ciencia do Esporte 2006; 27(3):169-183.

31. Ferreira VA, Magalhaes R. Nutrição e promoção da saúde: perspectivas atuais. Cad Saude Publica 2007; 23(7):1674-1681.

32. Castro ED, Silva DM. Atos e fatos de cultura: territórios das práticas, interdisciplinaridade e as ações na interface da arte e promoção da saúde. Rev Terapia Ocupacional 2007; 18(3):102-112.

33. Uchoa AC. Experiências inovadoras de cuidado no Programa Saúde da Família (PSF): potencialidades e limites. Interface (Botucatu)2009; 13(29):299-311.

34. Ischkanian PC, Pelicioni MCF. Challenges of complementary and alternative medicine in the SUS aiming to health promotion. Rev Bras Crescimento Desenvolv Hum 2012; 22(2):233-238.

35. Santos MC, Tesser CD. Um método para a implantação e promoção de acesso às Práticas Integrativas e Complementares na Atenção Primária à Saúde. Cien SaudeColet 2012; 17(11):3011-3024.

36. Felisberto E, Freese E, Bezerra LCA, Alves CKA, Samico I. Análise da sustentabilidade de uma política de avaliação: o caso da atenção básica no Brasil. Cad Saude Publica 2010; 26(6):1079-1095.

37. Bueno RE, Moysés ST, Bueno PAR, Moysés SJ. Governança, sustentabilidade e equidade no plano de saúde de São José dos Pinhais, Brasil. Rev Panam Salud Publica 2013; 34(6):416-421.

38. Mendonça MHM, Martins MIC, Giovanella L. Desafios para gestão do trabalho a partir de experiências exitosas de expansão da Estratégia de Saúde da Família. Cien Saude Colet 2010; 15(5):2355-2365.

Artigo apresentado em 02/02/2016

Aprovado em 07/04/2016

Versão final apresentada em 09/04/2016 
